\title{
Koloniale Konstellationen von Heimat und Fremde
}

\section{Wie Heimat und Fremde im Rückblick miteinander verschmelzen}

Für den deutschen Kolonialismus und auch die deutsche Kolonialliteratur sind Fremde und Heimat vielfach nichts anderes als zwei Seiten ein und derselben Medaille, so dass Faszination durch die Fremde und Heimatverbundenheit eng zusammenrücken. Exemplarisch deutlich machen kann man sich das an der Eingangspassage von Lydia Höpkers 1925 veröffentlichtem Südafrika-Roman Um Scholle und Leben. Schicksale einer deutschen Farmerin in Südwest-Afrika, in der die erwachsene Frau sich von Afrika aus an ihre Kindheit erinnert:

Sehnlichst wünschte ich mir als kleines Mädchen die Pantoffel des kleinen Muck. Sie standen sogar auf meinem Weihnachtswunschzettel. Dreimal mußte man sich auf ihnen herumdrehen und, hast du nicht gesehen, flog man fort in ein fernes, märchenhaftes Land.

An diese Wunderpantoffel musste ich denken, als ich eines Morgens auf Farm Kayas in den ersten Frühlingstagen im Ziegenkraal stand, eine ehrwürdige Ziegenmama zwischen den Beinen, ein Kaffernweib daneben mit einem kleinen Zicklein, diesem gut zuredend und ihm das volle Euter in das Maul stopfend.

(Höpker 1925, 5)

Faszination durch die Fremde in zeitlicher und alte deutsche Heimat in räumlicher Entfernung sind hier enger miteinander korreliert als die durch die Pantoffel des kleinen Muck ins Spiel gebrachte Sehnsucht nach der Fremde und der tatsächliche Aufenthalt in Südafrika. Anders formuliert: Die Faszination durch die Fremde wird nicht vom hic et nunc des Lebens in Afrika aus thematisiert, sondern in der gleich doppelten, zeitlichen und räumlichen Distanz der kindlichen Perspektive.

Eine ganz ähnliche Verknüpfung bzw. Parallelisierung von Fremde und Heimat findet sich auch in den unter dem Titel Mit Schwert und Pflugschar in Sachsen und Südwestafrika veröffentlichten Erinnerungen von Gottreich Hubertus Mehnert, einem Rittmeister der sogenannten Schutztruppe in Deutsch-Südwestafrika: 
In der am Wasser liegenden Sanddüne sind die Frösche erwacht, die mit dem untergehenden Strahl der Sonne ihr tausendstimmiges Quaken ertönen lassen.

Das rasselnde „Liebeslied“ der Frösche schlägt im Schlaf an mein Ohr, meine Jugendzeit steht vor mir auf: Am Strande der Elbe spielen wir Kinder [...]. Aus dem Weidenheger kommen die Spielverderber, mit dem Stock treiben uns unsere Gouvernanten nach Hause.

„Koffi!“ Das Buschmädchen ruft aus der Küche, raus aus dem Bett, hinein in die rauhe Wirklichkeit.

Wie ein Traum liegt das verflossene Leben hinter mir. Lasse ich aber des Morgens meine Kühe melken und der Bursche Saul ruft die Stammkühe Käthe, Hilmar, Lore, Ella und Margot in den Kral, dann kommt die Erinnerung an meine Jugendzeit, an das Elbdörfchen Amelgosswitz, wo ich am 26. November 1880 geboren wurde.

(Mehnert 2007, 15)

Zeigen diese beiden Beispiele der Überblendung von Heimat und Fremde, denen viele weitere an die Seite gestellt werden könnten, dass man als deutsche Kolonialistin oder deutscher Rittmeister in Afrika das Gefühl der Faszination durch die Fremde gar nicht artikulieren konnte? Und wenn ja, warum ist das so? ${ }^{1}$

Eine mögliche Erklärung liegt in den wechselseitigen Affinitäten von Kolonialismus und Heimatkunstideologie. Die deutsche Heimatkunstbewegung hatte nämlich - soweit sie sich als politisches Lebensreformprogramm zur Realisierung bzw. zur Rettung einer bedrohten Lebenswelt angesichts von Entfremdungserfahrungen verstand - auf heimischem Terrain um die Jahrhundertwende keine reale Chance mehr auf Verwirklichung ihrer traditionsorientierten Vorstellungen von Authentizität, Persönlichkeit, Volkstum und zyklisch-nachhaltigen agrarischen Lebensformen. Denn die exponentiell fortschreitende Industrialisierung mit ihren allerorten spürbaren Auswirkungen war längst irreversibel geworden. Ein Programmruf wie Friedrich Lienhards „Los von Berlin“ aus dem Jahre 1900 kann dafür als exemplarisch gelten.

Zeitlich parallel dazu sah sich der deutsche Kolonialismus vor die Aufgabe gestellt, Fremde in Heimat zu verwandeln, sich in den neuen Pacht-, Schutz- und Kolonialgebieten $\mathrm{zu}$,verwurzeln ${ }^{6}$ und die Kolonien dem deutschen Reichskörper politisch durch militärische bzw. paramilitärische Besetzung und diskursiv durch sogenannte ,Kultivation“ anzuschließen: „Bismarck-Archipel“ mit den Inseln „Neu-Pommern“, „Neu-Mecklenburg“ und „Neu-Hannover“ im heutigen Papua-Neuguinea; „Waldersee-Höhen“ und „Prinz-Heinrich-Berge“ im chinesischen Pachtgebiet Tsingtau; „Kaiser Wilhelmsland“ in Melanesien; „Bismarckburg“ in Togo; Farmen mit Namen wie „Deutsche Erde“ in Deutsch-Südwestafrika, dem heutigen Namibia. Fungierte die Fremde als Wohn-, Lebens- und agrarischer Wirtschaftsort dabei als ,neue Heimat‘ der kolonialen Ansiedler - gestützt durch die für

1 Dieser Beitrag greift passagenweise zurück auf Parr 2014. Ich danke dem Verlag für die Genehmigung zur Wieerverwendung in dieser überarbeiteten und erweiterten Fassung. 
die Kolonial- ebenso wie die Heimatkunstbewegung grundlegende Symbolik der ,Verwurzelung auf der eigenen Scholle، -, so stand die Aktualität der deutschen Heimat der Kolonialzeit dazu in latenter Opposition, wurde sie doch wegen der nicht mehr umzukehrenden industriellen Entwicklungen mit Befremdlichkeit konstituierender Distanz angeschaut.

\section{Wechselseitige Affinitäten zwischen Heimatkunst und Kolonialismus}

Vor diesem Hintergrund mussten Heimatkunst- und Kolonialbewegung und speziell noch einmal Kolonial- und Heimatkunstliteratur in der Zeit zwischen etwa 1885 und dem Beginn des Ersten Weltkriegs starke wechselseitige Affinitäten entwickeln, eröffneten die noch nicht industrialisierten Kolonialgebiete der Heimatkunstbewegung doch gleichsam eine zweite Chance, ihr Programm - wenn schon nicht mehr in Deutschland selbst - so doch zumindest in den Kolonien zu realisieren (vgl. Jäger 2009). Der suchende Blick nach heimatlicher ,Bodenständigkeit‘ konnte in die Kolonien umgelenkt werden, um dort in einem ersten Schritt das (wieder-) zu entdecken, was in Europa längst als obsolet gelten musste, und um es dann in einem zweiten Schritt auf dem Weg über die Kolonialliteratur und die vielgelesenen kolonialen Zeitschriften wieder in die alte Heimat zu re-importieren. Würde man nämlich aus der zitierten Stelle des Romans von Höpker die wenigen Afrikabezüge tilgen, so käme ein genuiner Text der Heimatkunstliteratur dabei heraus:

\footnotetext{
Sehnlichst wünschte ich mir als kleines Mädchen die Pantoffel des kleinen Muck. Sie standen sogar auf meinem Weihnachtswunschzettel. Dreimal mußte man sich auf ihnen herumdrehen und, hast du nicht gesehen, flog man fort in ein fernes, märchenhaftes Land.

An diese Wunderpantoffel musste ich denken, als ich eines Morgens auf unserem Hof in den ersten Frühlingstagen im Ziegenstall stand, eine ehrwürdige Ziegenmama zwischen den Beinen, eine Magd daneben mit einem kleinen Zicklein, diesem gut zuredend und ihm das volle Euter in das Maul stopfend.
}

Der koloniale Gang in die Fremde ist damit eigentlich eine Suchbewegung nach einer zeitlich bereits zurückliegenden Vorstellung von Heimat. Indem es nun galt, diese in der Fremde einzulösen, blieb kaum mehr eine Möglichkeit, ein sich auf Fremdes richtendes Interesse zu artikulieren. In der Terminologie der neueren Raumtheorien ließe sich sagen, dass im kolonialen Heimatkonstrukt ,space‘ (also semantisierter, mit ,Heimat'-Bedeutung aufgeladener Raum) und ,place‘ (empirischer geografischer Ort im südlichen Afrika) miteinander kurzgeschlossen werden. Das Zusammenspiel von Heimatkunst- und Kolonialbewe- 
gung bot damit um die Jahrhundertwende für einen Moment die Chance, die Diachronie von ,Erinnerung“, ,Status quo und ,Utopie‘ zu synchronisieren. Das, woran man sich im Mutterland nur noch erinnern konnte, sollte in den Kolonien zeitgleich zurückgewonnen werden, womit die retrospektive Blickrichtung der Heimatkunstbewegung und die prospektive der kolonialen Utopie tendenziell zusammenfielen.

\section{Formen des Umgangs mit dem auf Heimat hin ausgerichteten Kolonialismus}

Vier ganz verschiedene Beispiele und mit ihnen auch Modelle des Umlenkens bzw. des Umkodierens von Fremde in Heimat und damit auch von Faszination durch die Fremde in Heimatgefühle werden im Folgenden vorgestellt.

Erstens wird kurz auf die Burenkriege eingegangen, die eine attraktive Diskursposition für die deutschen Kolonialisten wie auch die deutsche Heimatkunstbewegung boten. Danach wird - zweitens - die Spezifik der Kopplung von militärisch-imperialem Kolonialismus und Heimatkunstideologie in Gustav Frenssens „Feldzugsbericht“ Peter Moors Fahrt nach Südwest (Frenssen 1906) analysiert, der den zwischen 1904 und 1908 gegen die Herero und Nama geführten deutschen Vernichtungskrieg thematisiert; dann - drittens - der Unauflösbarkeit der Aporie von ,Verheimatung، der Kolonien auf der einen und ihrer exotistischen Gefährdung auf der anderen Seite am Beispiel der 1913 erschienenen Südafrikanischen Novellen von Hans Grimm (1975) nachgegangen, die diesen Konflikt in immer wieder neuen, nur leicht abgewandelten und dadurch fast schon experimentell anmutenden Szenarien durchspielen. Gesucht wird darin nämlich nach - nicht nur semantischen - Positionen der Begegnung zwischen ,Schwarz und ,Weiß‘, die punktuell zwar zu gelingen scheinen, aber immer nur temporär Bestand haben, bevor auch sie der unhintergehbaren Aporie des imperialen deutschen Konstrukts von kolonialer Heimat zum Opfer fallen. Denn wenn es nicht nur um koloniale bzw. imperial-militärische Aneignung der Fremde, sondern um ,Verheimatung“ im Sinne gelingender „kulturelle[r] Grenz-Arbeit“ - so die Formulierung von Homi K. Bhabha - gehen soll, dann ist es für die „Begegnung mit der ,Neuheit““ unabdingbar, dass sie ,an dem Kontinuum von Vergangenheit und Gegenwart“ gerade nicht „teilhat“ (Bhabha 2000, 10). Das Heimatkonstrukt des deutschen Imperialismus mit seiner Projektion eines anachronistischen Heimatbildes auf die Kolonien verhinderte aber genau dies. Zum Abschluss wird viertens auf das Verhältnis von Faszination durch das Fremde und Rückkehr zur Heimat für die Expeditionsreisen der Zwischenkriegszeit eingegangen. 


\section{Die Burenkriege - eine attraktive Diskursposition}

August Julius Langbehn hatte das Paradox der kolonialen Verheimatung in Rembrandt als Erzieher schon in den 1890er Jahren erkannt und PreußenDeutschland die diskursive Position der Holländer anempfohlen, nämlich diejenige von „Seebauern“ (Langbehn 1982,147), eine Formulierung, mit der die semantische Diskrepanz zwischen ,horizontalem in die Ferne-Gehen' und ,Sesshaftigkeit' aufgefangen werden sollte. Ein wichtiges diskursives Ereignis, an dem diese Semantik dann vielfältig durchgespielt wurde und welches ein attraktives Diskursangebot gerade auch für Intellektuelle, Schriftsteller und der Heimatkunstbewegung nahestehende Künstler bot, waren die sogenannten Burenkriege zwischen 1899 und 1903, in denen England gegen die südafrikanischen Transvaalburen kämpfte. Die Buren - ihrerseits zugleich als ,deutsch“ und ,holländisch' semantisiert - nahmen dabei die Position der hochpositiv gewerteten, sesshaften Bauern ein, die ihre Heimat verteidigten, die Engländer diejenige einer in die Horizontalität der Ferne streifenden Nation, aber ohne ,Tiefgang' und ,Sesshaftigkeit'; in der Sprache der Zeit ohne ,Verwurzelung im Boden'. Ikonisch wurde etwa die Partisanentechnik der Buren als ein geradezu mystisches ,Wachsen aus dem Boden heraus‘ dargestellt (vgl. dazu die Beispiele bei Parr 2014, 58-65), demgegenüber die englischen ,Söldner` zunächst machtlos erschienen. Je stärker diese Diskursposition auch in Europa und speziell in Deutschland Verbreitung und Akzeptanz fand, umso mehr konnten sich davon angerufene Gruppen mit ihr (und den Buren) assoziieren. So schifften sich deutsche Förster in ihren Uniformen mit Fahrrad und Gewehr ein, um in Südafrika gegen England zu kämpfen; zahlreiche Literaten der Heimatkunstbewegung (und solche, die dafür gehalten wurden) engagierten sich im Burenhilfsbund (Karl May, Hermann Lingg), stellten ihre Textproduktion zumindest teilweise auf das Burenthema ab (Ludwig Thoma) oder fungierten als Annahmestelle für Spenden (Wilhelm Raabe).

\section{An der Schnittstelle von Heimatkunst und Kolonialismus: Gustav Frenssens Peter Moors Fahrt nach Südwest}

Ebenfalls an der Schnittstelle von Heimat- und Kolonialliteratur platziert ist thematisch Gustav Frenssens 1906 erschienener Roman Peter Moors Fahrt nach Südwest (vgl. dazu Parr 2003; Detering und Sina 2018), und zwar bereits deutlich bevor eine Schriftstellerin wie Friederike Henriette Kraze die beiden ,Heimaten“ im Titel ihres 1909 erschienenen Romans Heim Neuland. Ein Roman von 
der Wasserkante und aus Deutsch-Südwest nebeneinanderstellte. ${ }^{2}$ Ein Autor aus der niederdeutschen Provinz, der sich mit Romanen wie Jörn Uhl (1901) und Hilligenlei (1905) als Vertreter einer norddeutschen Heimatkunstliteratur einen Namen gemacht hatte, schrieb 1906 plötzlich über die Kolonien, genauer über einen jungen Mann niederdeutscher Herkunft, der als Marineinfanterist nach Deutsch-Südwestafrika kommt, im Vernichtungskrieg gegen die Herero und Nama eingesetzt wird und nach etlichen Fremdheitserfahrungen und körperlichen Strapazen wieder zu Hause anlangt. Dieser Feldzugsbericht wurde von der zeitgenössischen Kritik in Deutschland nahezu einhellig positiv aufgenommen und der Erfolg vielfach durch den „Volkston“ bzw. den Charakter eines „Volksbuches“ (vgl. die Belege bei Benninghoff-Lühl 1983, 134) erklärt, wobei dem „derben, duftenden Bauernbrot“ heimatkünstlerischen Erzählens ganz im Sinne der Heimatkunstbewegung das negativ gewertete „,aufrasende Maschinenzeitalter“ und die Großstadt als dessen Sinnbild entgegengestellt wurde (so Jansen 1933, 15-16 [zit. nach Warmbold 1982, 97], zu Jörn Uhl und Hilligenlei).

Ein Problem für die Autor^innen der Heimatkunstbewegung war jedoch, dass sie dann, wenn sie als regionale Schriftsteller^innen wahrgenommen wurden - was dem Heimatkunstprogramm eigentlich entsprochen hätte -, zugleich in der Gefahr standen, auch nur regional rezipiert zu werden. Unter ökonomischem Aspekt mussten sie es daher schaffen, die Verbundenheit mit der per se regionalen, wenn nicht sogar lokalen ,Scholle“ ins ,AllgemeinDeutsche‘ zu wenden, was um 1900 stets auch hieß: ,ins Koloniale‘. Hatte Frenssen in seinem 1901 erschienenen Roman Jörn Uhl dieses Problem auf einem eher indirekten Wege noch dadurch zu lösen gesucht, dass er Wissen aus und um die Kolonien in Berichtsform punktuell auch in der norddeutschen Heimat verfügbar machte (231-242), so gelang es ihm mit Peter Moors Fahrt vom regionalen zum gesamtdeutschen Schriftsteller zu avancieren (vgl. Benninghoff-Lühl 1983, 57; Detering und Sina 2018, 10). Denn die mit Peter Moor thematisierten Kolonien waren ein Gegenstand, an dem sich die Basisideologeme und -optionen der Heimatkunstbewegung auch direkt in globaler und nicht nur regionaler Perspektive umsetzen ließen. Das Interesse an dem dann nur noch auf den ersten Blick überraschenden Thema Südafrika war also auch durch die der Heimatkunstbewegung interne Logik motiviert, wobei allerdings immer die Rückbindung an das Heimatland gegeben sein musste.

2 Ketelsen 2006 betont, dass Krazes Roman „in derselben Weise ein Kolonialroman ist, wie er ein Heimatroman ist“ (138), ein Roman, „der so offenkundig gemäß den diskursiven Regeln des Heimat- und des Kolonialromans verfaßt worden ist“ (145). 
Die Materialbasis für seinen Text bezog Frenssen aus Briefen, Tagebüchern, Bildern, kriegsgeschichtlicher Literatur und nicht zuletzt von den zurückkehrenden Soldaten, die er ausführlich interviewte (siehe Benninghoff-Lühl 1983, 124-125) - ein aus Sicht der Ästhetik der Heimatkunstbewegung eigentlich grenzwertiges, weil schon in Richtung „Reportageroman“ (Meyn 1997, 324) verweisendes Verfahren der Informationsbeschaffung. Das warf jedoch wiederum das Problem auf, dass damit jene Erdverbundenheit gefährdet war, ohne die ein Schriftsteller der Heimatkunstbewegung ihrer Programmatik nach gar nicht erst hätte schreiben können. Frenssens Lösung, die er zur Rechtfertigung später explizit formulierte, lautete: „Ich hatte nach so viel Lektüre, soviel Grübeln und geistigem Sehen, so viel genauer Befragung nicht mehr das Gefühl, daß ich nicht Selbstgesehenes und Erlebtes darstellte. Ich hatte den Sand nun zwischen den eigenen Zähnen.“ (Frenssen 1909, 5; vgl. Benninghoff-Lühl 1983, 124; Warmbold 1982, 101) Fremderfahrung wird hier in quasi-authentische Eigenerfahrung überführt, das ferne Afrika in eine in den Anschauungsformen der Heimatkunstbewegung artikulierte Unmittelbarkeit.

Deutlich wird damit, dass Frenssen keinen exotistischen „Reiseroman“ (Warmbold 1982, 102) schreiben, nicht exakte Informationen liefern wollte, mit denen spätere Leser die Schauplätze hätten abwandern können, sondern vielmehr darauf abzielte, ästhetisch-ideologische Optionen umzusetzen, die die Heimat (und Heimatkunst) in Deutschland betrafen. Diese Richtung des Interesses wird zunächst durch die Perspektive der Hauptfigur hergestellt: Es geht um einen „,holsteinische[n] Jungen aus Handwerkerhaus“, der auf kolonialdeutschem Boden für gesamtdeutsche Ideale“ (Warmbold 1982, 102) - und nicht nur solche regionaldeutscher Heimatkunst - streitet und sie mit seinem Kampf zumindest ansatzweise zu verwirklichen hilft. Daher ist im Roman eine Tendenz zu beobachten, bei Aufzählungen stets das ganze Deutschland von Norden nach Süden, von Westen nach Osten in seinen regionalen Landsmannschaften (man könne auch sagen: in seinen Heimaten) zu repräsentieren: „Schlesier und Bayern und aus allen andern deutschen Stämmen, und auch drei oder vier Holsteiner.“ (Frenssen 1906, 6)

Sobald Frenssen aber von deutschen Farmen oder Siedlungen schreibt, wird das ganze Repertoire an Boden-, Garten-, Bauern- und nicht zuletzt Familienideologie der Heimatkunst aufgeboten, und zwar so, als hätten die kolonialen Aktivitäten immer schon genau darauf abgezielt. Selbst dem verwüsteten, „schmalen und dürftigen Garten“ einer Ansiedlung sieht man noch an, „mit welcher Mühe deutsche Hände ihn in dem dünnen Erdreich gepflegt hatten“ (Frenssen 1906, 41; vgl. dazu Warmbold 1982, 113-114, 119); Farmhäuser sind stets „schön und stattlich“ (Frenssen 1906, 50) und weiße reinliche Frauen erscheinen als deutsche Madonnen: 
Mit großen Augen spähten wir in den Garten hinein, den die Schutztruppler in früheren Jahren mit großer Mühe hier angelegt hatten; da waren wahrhaftig Palmen und Weinlauben, von denen wir in Kiel und auf dem Meere geträumt und geredet hatten; und da war ein Teich! O, wenn man da hineinreiten dürfte! Und da, im Schatten einer Veranda, stand eine deutsche Frau; sie hatte ein kleines Kind auf dem Arm. Wie wir hinsahen! Wie wir uns über das helle, saubere Kleid freuten und über das reine, freundliche Gesicht und über das kleine weiße Kind. Wie auf ein Himmelswunder starrten wir auf das, was man in Deutschland alle Tage sehn konnte. Wie die heiligen drei Könige, die auch aus der Wüste kamen und vom Pferd herab Maria mit ihrem Kinde sahen. Sie sah uns hungrige, ganz verlumpte und schmutzige Gesellen mit großen, mitleidigen Augen an und neigte sich freundlich, als wir alle wie auf Befehl die Hand an die Feldmütze legten.

(Frenssen 1906, 111)

Zur Mitte des Romans hin wird das Heimatkunstprogramm dann breit entfaltet, und zwar als etwas, das in Deutschland gegen die Industrialisierung auch schon zum Zeitpunkt des Erscheinens von Frenssens Roman so nicht mehr zu verwirklichen war. Einen älteren Kolonnenführer fragt Peter Moor „wie er es anfange, eine Farm zu gründen“. Dessen Antwort umreißt die von der Heimatkunstbewegung popularisierte Imagination eines ,besseren` Deutschland jenseits von Untermietern, Tagelöhnern, Leistungskonkurrenz und Mangel an Raum:

„Ich suche mir einen Platz aus mit gutem Wasser und guter Weide; dort lasse ich mir von der Regierung so ungefähr fünftausend Hektar anweisen. Es geht nicht so genau wie in Deutschland, sondern es heißt: von dem Baum bis zu dem Wasserloch, und dann zu der Pad, und so weiter. Dann lasse ich das bißchen Vieh, das ich habe, dort weiden. Es nährt und tränkt und mehrt sich selbst, ganz wie bei Abraham und Jakob. Nach zwei, drei Jahren habe ich schon eine ganze Herde. Unterdes baue ich mir ein kleines steinernes Haus. Wenn ich allmählich anfange, einige Stücke Vieh zu verkaufen, wird aus dem Haus ein besseres.“ Ich fragte ihn, ob er trotz des Aufstandes und all der Zerstörung im Lande bleiben wolle. Er sagte: „Sieh! Du kannst hier gehn und stehn und ruhn und trekken, hundert Meilen, und kein Mensch sagt Dir, was Du sollst oder nicht, und Du hast keine Sorge um Freundschaft mit dem Nachbar auf derselben Etage, oder mit dem Vizewirt um die Tapete im Wohnzimmer, oder um Tagelohn, oder um täglich Brot. Wenn Du das eine Kalb verzehrt hast, schlachtest Du ein andres. Magst Du kein Kalbfleisch mehr, schlachtest Du eine Ziege. Oder Du gehst auf die Jagd [...]. “ Ich fragte ihn, ob er wohl heiraten wolle. Er sah mich von der Seite an und sagte: „Wenn der Krieg zu Ende ist, kommt ein Mädchen aus Deutschland, mit dem ich brieflich eins geworden bin. Ich kenne ihre Eltern und auch sie ein bißchen. Die Farmerfrauen sind hier guter Dinge, das kannst Du Dir auch denken; wenig Arbeit, keine Konkurrenz, also kein Neid und Streit, viel Land, Kühe und Ochsen, ein Pferd zum Reiten, keine Sorge ums Auskommen. "So erzählte er. Ich hörte ihm gern zu, und konnte alles, was er sagte, wohl verstehn.

(Frenssen 1906, 124-126; vgl. dazu auch Kiesel 2018, 32)

Folgt man dem, dann gibt es eigentlich nur noch einen Störfaktor bei der Verwirklichung des Heimatkunstprogramms - nämlich die Afrikanerinnen, die dann nicht nur kriegskonsequent, sondern auch konsequent im Sinne der Heimatkunstbewegung ausgerottet werden. Auch wenn man denken könnte, dass 
das auf der Folie der christlichen Grundannahme ,alle Menschen sind Brüder` für die Kolonialist*innen zunächst einmal durchaus problematisch war, konnte ein solcher Rassismus auf dem Feld der Religion doch mit der Heimatkunstideologie verknüpft werden. Am Ende von Frenssens Roman wird nämlich ein Feldgottesdienst geschildert, der Gelegenheit gibt, das christliche Gebot der brüderlichen Gleichheit zum einen rassistisch, zum anderen mit Blick auf die Basisannahmen der Heimatkunstbewegung $\mathrm{zu}$ interpretieren. Es dient zum einen der trivialdarwinistisch-rassistischen Legitimation des Genozids an den Herero und Nama:

Was wir vorgestern beim Gottesdienst gesungen haben [...], das verstehe ich so: Gott hat uns hier siegen lassen, weil wir die Edleren und Vorwärtsstrebenden sind. Das will aber nicht viel sagen gegenüber diesem schwarzen Volk; sondern wir müssen sorgen, daß wir vor allen Völkern der Erde die Besseren und Wacheren werden. Den Tüchtigeren, den Frischeren gehört die Welt. Das ist Gottes Gerechtigkeit.

(Frenssen 1906, 200)

Zum anderen geschieht diese Rechtfertigung aber mit Bezug auf die Ideologeme der Heimatkunstbewegung: „Diese Schwarzen haben vor Gott und Menschen den Tod verdient, nicht weil sie die zweihundert Farmer ermordet haben und gegen uns aufgestanden sind, sondern weil sie keine Häuser gebaut und keine Brunnen gegraben haben.“ (Frenssen 1906, 200; zum Argumentationsmuster vgl. Brehl 2004) Ganz konsequent beginnt das letzte Kapitel des Romans dann auch damit, dass in dem Moment, in dem die Herero besiegt und Südafrika in deutscher Hand ist, die Landschaft unter dem einsetzenden Regen zu sprießen, zu wachsen, zu blühen beginnt und sich damit Fremde erfolgreich in Heimat verwandelt hat. ${ }^{3}$ Das Heimatkunstprogramm wird damit bei Frenssen auf dem Weg über den Krieg gegen die Herero in Südafrika, also gleichsam out-of-area, verwirklicht (vgl. Brehl 2000).

\section{Exotistisch gefährdete Heimaten: Hans Grimms Südafrikanische Novellen (1913)}

Ein drittes, ganz anders strukturiertes und weitaus komplexeres Modell des Umgangs mit der in der deutschen Kolonialliteratur offenbar tabuisierten Artikulation von Faszination durch die Fremde entwickeln Hans Grimms Südafrikaerzählungen. Werden bei Frenssen die relativ einfach strukturierten Leitoppositionen

3 Vgl. auch Malherbe-Boucke 1936, 67: „Der Boden bleibt arm; was aber menschenmöglich war, ist ihm abgerungen. Die einst nutzlosen großen Flächen sind längst Kapstadts Gemüse- und Kartoffelgärten geworden. Ihre Erfolge haben das rühmende Wort geprägt: ,Auf einem Boden, auf dem Schotten und Engländer verzagen, auf dem nicht genug für die Schildkröte wächst, da schaffen Deutsche noch ein Paradies.“” 
von ,schmutzig vs. rein‘, ,Einzelne vs. Masse‘, ,sesshaft vs. nomadisch“, ,Nord vs. Süd‘, ,Wasser vs. Trockenheit‘ kaum durch exotistische Faszinationen irritiert, da dies zu vielfältigen Friktionen hätte führen müssen (lediglich einmal ist die Rede von „Kameraden“, die „wollten, dass Afrika ganz, ganz anders aussähe als die Heimat“, Frenssen 1906, 44), so spielt genau diese Faszination in den 1913 veröffentlichten Südafrikanischen Novellen von Hans Grimm eine besondere Rolle. Damit werden diejenigen semantischen Elemente von besonderem Interesse, die geeignet sind, ,Heimat und Fremde‘, ,Kolonialismus und Exotismus‘ zu vermitteln, sie koexistieren zu lassen.

Während Frenssens Konzept eines zugleich biologistisch, religiös wie auch heimatkünstlerisch argumentierenden Rassismus solche Berührungen aber gar nicht erst zuließ, spielt Grimm in den Zonen des Überlappens der semantischen Paradigmen von ,Schwarz‘ und ,Weiß‘ verschiedene Szenarien des Scheiterns eines dauerhaften Zusammenlebens durch. Dabei geht es Grimm sicherlich auch darum, sein „Konglomerat aus Tropenhygiene und Biopolitik“ sowie einer „rassistischnationalkonservativen Weltsicht“ und Ideologie „möglichst effektvoll und umfassend zu vermitteln“ (Freudenthal 2010, 24), doch werden die Zonen des Berührens und Überlappens von Schwarz und Weiß immerhin probeweise durchgespielt, was für einen großen Teil der Kolonialliteratur, wie nicht zuletzt das Beispiel Frenssen zeigt, gar nicht im Horizont des Denkbaren liegt.

Thematisiert wird bei Grimm damit auch hier wieder ein Bedingungsdreieck von Exotismus (Faszination an der Fremde), Imperialismus und Heimat, und zwar in einer spezifisch südwestafrikanischen, das heißt deutsch-kolonialen Variante.

\section{„Ferngewohnheit“: Das ,Braune“ zwischen ,Schwarzem‘ und ,Weißem“ in der Novelle Dina}

Hans Grimms Novelle Dina (Grimm 1975, 7-41), an der sich das exemplarisch zeigen lässt, spielt nur kurze Zeit nach dem bei Frenssen behandelten Krieg gegen die Herero. Der namenlos bleibende Wachtmeister einer Polizeistation in der Lüderitzbucht trifft auf einer seiner Inspektionstouren die Hottentottin Dina und einen sie begleitenden, etwas verwachsenen Jungen an einem Wasserloch. Er nimmt beide mit zur Station, auf der sich Dina auf vielfältige Weise nützlich macht, im Wachtmeister aber auch ein erotisches Begehren weckt - und zugleich auch ein damit nicht per se gleichzusetzendes exotistisches Begehren befriedigt.

Dieser Konstellation entsprechend, verweisen die semantischen Eckpole der Erzählung von diesem mittleren Feld des Zusammenkommens von Schwarz und Weiß zum einen deutlich in Richtung ,Afrika', zum anderen ebenso deutlich in Richtung ,Deutschland' bzw. ,Holstein'. Die sich daraus ergebende gleichermaßen 
semantische wie geografische Achse stellt ein Kontinuum zwischen ,Schwarz und ,Weiß‘, zwischen ,Lüderitzbucht‘ und ,Holstein“ dar, innerhalb dessen die Hauptfiguren platziert werden. Relativ schnell kristallisieren sich dabei über die ihnen zugeschriebenen Attribute distinkte Positionen heraus, unter denen ganze Paradigmen von sich wechselseitig stützenden und verstärkenden semantischen Elementen subsumiert sind, z. B. ,schwarz, kindisch, faul, tierisch, warm-trocken“ versus ,weiß, erwachsen, fleißig, menschlich, ordentlich, kalt-nass‘.

Zwischen diesen Extremen gibt es einen Zwischenbereich, in dem die polaren Merkmale punktuell und temporär , auf die andere Seite‘ überwechseln können, sodass der Zwischen- zum Vermittlungsbereich wird. ,Ordentlichkeit‘ etwa ist ein zunächst durchgehend an die deutschen Figuren gekoppeltes Merkmal. Die Frau des Wachtmeisters wird als „Norddeutsche [...] mit der niedersächsischen Ordentlichkeit im Leibe“ beschrieben; die Deutschen überziehen selbst „tote[s] Land“ mit einem „lebendige[n] Netz von Ordentlichkeit“, sodass „Propertät“ herrscht. Ordentlichkeit ist aber auch ein Merkmal, das - mit Einschränkung - der Hottentottin Dina zugesprochen wird: „[...] sie hat keine Läuse und ist überhaupt sehr reinlich.“ (Grimm 1975, 7, 14, 16) Weiter wird sie charakterisiert als „ordentlich gewachsen“ (erfüllt also ein deutsches Kriterium), aber „nach ihrer Art“ (was sie wiederum relativierend innerhalb von Afrika verortet). Auf diese Weise kann sie eine ambivalent zwischen Heimat und Fremde bzw. Kolonialismus und Exotismus changierende Mittelposition einnehmen. Der Dina begleitende Hottentottenjunge hat im Gegensatz zu ihr einen „Höcker“, fast so, als sei das der Normalfall, eine „ganz ordentlich gewachsen[e]“ (Grimm 1975, 9) Dina jedoch die in Richtung europäische Normalität tendierende Ausnahme. Dina rückt damit punktuell über die Mittelposition hinaus und der Tendenz nach in den semantischen Bereich ,Deutschland', speziell ,Holstein/Heimat' hinein.

Umgekehrt sucht der deutsche, ,braungebrannte“ und von „Ferngewohnheit“ (Grimm 1975, 19; Hervorhebungen R.P.) getriebene Wachtmeister ganz so wie der afrikanische Landstreicher immer die ,Weite‘. Das wiederum steht ganz im Gegensatz zu seiner weißen Frau, die ständig Heimweh und Sehnsucht nach der ,Enge، der Heimat artikuliert und bereits auf dem Dampfer vor Southampton erkennt, „daß sie außerhalb der Heimat nie werde Wurzel fassen können, was sonst immer sich ereignen möge“ (Grimm 1975, 19). Das aber stellt nicht nur den denkbar gröbsten Verstoß gegen die koloniale Aufgabe der Verheimatung von Fremde dar, indem eine Differenz zwischen geopolitischer ,deutscher Heimat' und geokultureller bzw. semantischer Fremdheitserfahrung aufgemacht wird, sondern bringt den Wachtmeister obendrein in maximale Distanz zu seiner ,richtige[n] weiße[n] Frau“ (Grimm 1975, 19): „So stark wie in dem Holsteiner die Ferngewohnheit war, so groß war in ihr das Heimweh.“ (Grimm 1975, 19) Die weiße Frau verkörpert also die negative Variante der Faszination durch das Fremde, nämlich ein Heimweh, 
das sich auf die aktuelle Heimat richtet und nicht die Vorstellung der besseren, aber vergangenen Heimat, die es in Afrika einzulösen gilt.

Bereits im allerersten Absatz der Erzählung werden auf ganz engem Raum Deutschland bzw. Holstein und Südafrika aufeinander bezogen und gleichsam probeweise miteinander verbunden, indem einzelne semantische Merkmale auf beiden Seiten verortet werden. So hört der Wachtmeister aus dem „Kauderwelsch“ der Hottentottin den Namen „Dina“ heraus, gleichzeitig hört er ihn aber auch aus dem Niederdeutschen heraus: „Sie nannte sich gar nicht Dina, aber dem Wachtmeister der Polizeitruppe, der sie fand und fragte, klang es ähnlich aus dem Kauderwelsch heraus, und von seiner Schwägerin daheim in Holstein war er an den Namen gewöhnt.“ (Grimm 1975, 7) Damit ist Dina wiederum auf der Mitte zwischen Heimat und Fremde platziert: Sie wird gleichzeitig in maximale Distanz gestellt und nah herangerückt.

\section{Koloniales versus exotistisches Begehren}

Die ,weiße Frau' ist bei Grimm (und darüber hinaus einem großen Teil der Kolonialliteratur) - wie Peter Horn (1985) überzeugend nachgewiesen hat - sexuell eher neutral, ohne erotische Ausstrahlung. Dadurch ist sie in gleich mehrfacher Hinsicht gerade nicht ,Dienerin“ ihres Mannes, sondern koloniale ,Herrscherin“ und ,Fürstin“ neben ihm. Als eine solche ,weiße Herrin‘ verlässt die weiße Frau ihre typische weibliche Geschlechtsrolle als Dienerin. Diese Funktionsstelle ist in Grimms Erzählung zu Beginn frei und kann von Dina wahrgenommen und besetzt werden. Das bedeutet aber auch: Indem die weiße Frau ihre Geschlechtsrolle verlässt, gefährdet sie die koloniale Ordnung ebenso, wie sie zugleich die Verbindung des Kolonialisten mit der farbigen Frau fördert.

Zwischen farbiger und weißer Frau steht der deutsche Wachtmeister, für den das exotistisch begehrte Paradies zu einem vergifteten Ort wird. Denn der Kolonist, der ein Paradies, also das, worauf sich seine „Ferngewohnheit“ richtet, der ,Freiheit“ und Ungebundenheit begehrt, steht zugleich in einer Funktion, in der er all dies seinem ,mitgebrachten deutschen Gesetz‘, dem „Netz von Ordentlichkeit“ (Grimm 1975, 14) unterwerfen muss, um als Kolonist überhaupt überleben zu können. Daraus entspringt der eigentliche Konflikt des Wachtmeisters: Dina gefährdet nicht sein exotistisches, auf die Fremde bezogenes, wohl aber sein koloniales und damit auf Heimat gerichtetes Begehren. Umgekehrt die weiße Frau: Sie gefährdet sein exotistisches, nicht aber sein kolonial-heimatliches Begehren. Überschreitet der Wachtmeister die Grenze zu Dina, dann vernichtet er sich als Kolonialherr, kann aber Exotist bleiben. Bleibt er ein die deutsche Heimat behauptender Kolonialherr, so muss er dieser Position sein exotistisch-sexuelles Begehren unterordnen. 
Die Erzählung spielt also die Unmöglichkeit eines gelungenen halben Weges, einer gelungenen Besetzung des Zwischenraums zwischen ,Schwarz' und ,Weiß‘, zwischen ,Fremde‘ und ,Heimat‘, zwischen kolonialer Verwurzelung und „Ferngewohnheit“ durch. Vermittlungen der jeweils antagonistisch angelegten Pole sind dabei immer nur temporär, nicht aber auf Dauer möglich, sodass es auch kein dauerhaft lebbares Modell zwischen ,Holstein“ und ,Südafrika' gibt, keine Kolonie als Heimat, sondern immer nur ein Umsteuern von dem einen als defizitär empfundenen Zustand, nämlich zu Hause zu sein in der nicht verheimateten und zudem auch noch als exotistisch rezipierten Fremde, in den anderen, nicht minder defizitären Zustand, nämlich Fremder in der als zu eng empfundenen Holsteinischen Heimat zu sein: „[...] auf einmal saß der Wachtmeister schon sechs Wochen in Holstein. Nach diesen sechs unklaren Wochen merkte er immer deutlicher, daß er ein Fremder sei in der Heimat.“ (Grimm 1975, 17) Dort

\begin{abstract}
erzählte er nicht nur dann und wann von Afrika, sondern beim kleinsten Anlasse holten ihn seine Gedanken fort über See, und dann schraubte er die Augen zusammen, ganz eng, wie einer, der bei grellem Sonnenglanze oder starkem Wind reitet und über weite Flächen scharf ausspähen muß. Die so eingestellten Augen sahen gar nicht mehr in die saftigen grünen Koppeln Holsteins hinein, die starrten in die unbezähmbare Ödheit der Namib.
\end{abstract}

(Grimm 1975, 17)

Grimms Erzählung zielt damit darauf ab, genau das zu desillusionieren, was Zeitschriften wie Kolonie und Heimat für ihr heimatliches und kolonial-heimatliches Lesepublikum semantisch kunstvoll in Szene setzten. Mit der Kollision von ,Heimat‘ und ,Exotismus“ bzw. von ,Heimat" und „Ferngewohnheit“ entzieht Grimm aber auch einer um Elemente der Heimatkunstbewegung ergänzten werbend-agitatorischen Kolonialliteratur weitestgehend den für sie eigentlich so nötigen diskursiven ,Heimatboden'. Das wiederum zeigt, wie prekär jede Form von ,Sich-Einlassen-auf-dasFremde‘ für den deutschen Kolonialismus sein musste.

\title{
Ausblick: Das Modell der Expeditionsreisen der Zwischenkriegszeit
}

Ein viertes, noch einmal anders akzentuiertes Modell der Verknüpfung von Fremde und Heimat und - diesmal auch durchaus emphatisch - Heimweh stellen die Expeditionen der Zwischenkriegszeit dar, denn sie folgen einem ganz anderen Bewegungskonzept als das koloniale Modell. Allerdings konnten die nach dem Ersten Weltkrieg verstärkt unternommenen Expeditionsreisen und die mit ihnen verbundenen symbolischen Inbesitznahmen der Fremde diskursiv als Ersatz für die mit 
dem Vertrag von Versailles endgültig verlorenen Kolonialgebiete dienen, sodass man von einer quasi-kolonialistischen Überdetermination der Expeditionen sprechen kann, teils in Kombination mit sportiv semantisierten Narrativen nationaler Konkurrenz. Nicht ersetzen ließ sich auf diese Weise aber das koloniale Modell dauerhafter Verheimatung in der Fremde.

Denn Expeditionen müssen den Raum, den sie erkunden wollen, unabhängig vom tatsächlichen Stand der Kenntnisse, als einen des Nicht-Wissens konzipieren, als einen jener weißen Flecken auf der Landkarte, die es von Nicht-Wissen in Wissen zu überführen gilt. Das wiederum ist nur im Erst-Fall möglich, also bei Ersterkundungen, -beschreibungen, -vermessungen, -besteigungen, -durchquerungen und seit den 1920er Jahren vermehrt durch Erstverfilmungen, was nichts anderes heißt, als Erst-Sichtbarmachung des Fremden in der Heimat. Die dann in der Regel nach den Expeditionen als deren eigentliche Ergebnisse produzierten illustrierten Bücher und Kinofilme, die meist stärker literarisch als wissenschaftlich angelegt sind, beschreiben häufig diese Überführung von Nicht-Wissen im Sinne von ,Noch-nichtGesehenem' in Wissen (Sichtbares). Ist dies der eine Bezug der Expeditionsreisen auf die Heimat, so liegt ein zweiter darin, das ,Auslandsdeutschtum“, das durch den Verlust der Kolonien in einem vielfach nicht nur übertragenen Sinne heimatlos geworden war, wieder an das ursprüngliche Heimatland anzuschließen.

Obwohl die Bewegungsform der Expedition also eine ,in die Weite der mehr oder weniger fremden Welt' ist, ist sie doch zugleich in zweifacher Weise auf die Heimat bezogen. Spätestens damit aber wirken die durch Expeditionen punktuell erschlossenen neuen Räume des Wissens auch auf die Ausgangskultur (im emphatischen Sinne auf die Heimat) zurück, handelt es sich bei ihnen doch um solche Räume, die sich als Konkretisationen des Wissens um Auslandsdeutsche darstellen. Verstärkt wird dies noch einmal, wenn in den bereisten Gebieten auf Zeugnisse des deutschen Kolonialismus gestoßen wird, so dass das koloniale Heimatkonstrukt mit dem der Expeditionen punktuell verknüpft werden kann. Von den Expeditionsreisen mit in die Heimat gebracht wird dann ein gleichsam verifiziertes Wissen um die offensichtlichen Erfolge deutscher kolonialer Verheimatung. Das verschafft einerseits dem deutschen Kolonialismus nachträgliche Legitimation, andererseits aber auch den Expeditionsreisen, die mit ihren Erkundungen der Fremde dann stets auch Erkundungen der Heimat sind.

Letztlich stellt sich die Expeditionsfahrt damit zugleich als eine aus der Heimat in die Fremde und als eine aus der alten Heimat in die vormals neue Heimat dar, nämlich zu den ,Auslandsdeutschen'. Sie werden nach der Unterbrechung durch den Ersten Weltkrieg in Form der beide verbindenden Reise wieder an die alte Heimat angeschlossen. Ein eigentlich vorhandenes, zwischenzeitlich aber $\mathrm{zu}$ verschwinden drohendes Wissen um die ehemaligen deutschen Kolonien und Siedlungsgebiete wird so in die alte Heimat zurückgeholt, womit der 
nach Versailles nicht mehr gegebene politisch-staatsrechtliche Anschluss der Kolonien durch einen diskursiven ersetzt wird. In dieser Situation kann dann die Faszination durch das Fremde wieder artikuliert werden, stellt es sich im Bewegungsmodell der Expeditionsreisen doch immer auch als doppelt abgesichertes Heimweh dar: In der Ferne trifft man auf das Auslandsdeutschtum, nach Deutschland zurück bringt man das Wissen darum.

Illustrieren lässt sich das an Günther Plüschows Expeditionsbericht Segelfahrt ins Wunderland von 1926, in dem er immer wieder auf erfolgreiche Projekte deutscher Kolonisation und Verheimatung verweist: Kaum blüht und sprießt es irgendwo, kaum gibt es prosperierende Farmen und besonders saubere Häuser und Tische, dann sind es deutsche Kolonisten mit ihren ,deutschen Tugenden', auf die das zurück geht. So erscheint die chilenische Stadt Valdivia als eine „schöne, gepflegte, schmucke Stadt“, ja geradezu als ein Hort „des festesten, treuesten Deutschtums, deutscher Arbeit und Kraft, deutscher Gastlichkeit“:

So rein und klar hat sich das Deutschtum, die deutsche Sprache hier erhalten, wie sonst nirgends in der Welt im Auslande, ja selbst noch der alte Heimatdialekt klingt durch.

$[\ldots]$

Da geht mir das Herz auf vor Stolz und Glück, ich kann bald aus vollster Überzeugung denken und sagen: Wenn in Deutschland die Deutschen selbst so treu und unerschütterlich zur Heimat ständen, so deutsch, wahrhaft deutsch dächten - es stände besser um unser geliebtes Vaterland.

(Plüschow 1926, 42-43; vgl. auch 59-60 und 80)

Das Wissen, das Expeditionsreisen wie die von Plüschow mit nach Hause bringen, ist damit auf zwei Ebenen angelegt. Zum einen ist es eines, das eigentlich aus der Heimat stammt, auf jeden Fall aber eines über und für die Heimat ist. Dieses Wissen macht die bereisten Gebiete auf dem Weg über das ,Auslandsdeutschtum‘ zu einem exponierten Glied des heimischen Staatskörpers, und zwar ganz analog zu den nach 1919 verlorenen Kolonien. Dieses Wissen dient dazu, der Heimat zu versichern, dass es das, was mit den Kolonien verloren ging, auf andere Art und Weise noch gibt. Dazu aber mussten die Orte, an denen man auf das ,Auslandsdeutschtum traf, zunächst in die Ferne gestellt werden, mussten als Gegenden konzipiert werden, in denen es Neues zu entdecken oder Bekanntes medial neu darzustellen gab.

Für ein und denselben geografischen Raum werden auf diese Weise also zwei ganz verschiedene Formen von Wissen in die Heimat transferiert: eines um das Eigene in der Fremde und eines, das sich als medial neues Wissen - und bisweilen auch nur medial neue Aufbereitung eines in anderer Form längst vorhandenen Wissens - darstellt. Von daher ist die von Plüschow in Segelfahrt ins Wunderland gestellte Frage „Weiß das deutsche Volk, daß hier im fernen Lande das Deutschtum stolz und stark und treu zur alten Heimat steht?“ (Plüschow 1926, 153-154) alles andere als bloß rhetorischer Art. Bedingung dafür wiederum ist, 
nach einer Expeditionsreise wieder in der Heimat anzukommen. Von daher steht am Ende der populären Berichte der in der Regel beschwerliche Weg zurück, der mindestens so wichtig ist wie die Explorations-Bewegung in die Weite der Welt.

Auch Plüschow beschreibt das Ende seiner ersten Expeditions-Reise als hindernisreiches ,Sich-Durchschlagen-in-die-Heimat' gegen die Unbill der See und des Wetters, dem zu Beginn der zweiten Reise das mühsame Kämpfen um Ausrüstung und Finanzierung korrespondiert (vgl. Plüschow 1929, 15). Die Rückkehr in die Heimat nach der Exploration der Fremde wird am Ende des Textes dann in gleich mehrfacher Überbietung des je schon Gesagten als erfolgreiche Beendigung der Explorations-Bewegung pathetisch inszeniert:

Wir an Bord sind voller Zuversicht. Zwar kommen wir nicht so schnell vorwärts, wie wir es hofften und wünschten, der Sturm hält uns zurück, aber langsam und sicher dringen wir kämpfend und ringend gen Osten vor. Das ist ja das Schöne, das Befreiende, die Gewißheit: Wir siegen, die Heimat winkt.

Und an einem strahlenden Morgen steigt die deutsche Küste aus dem Meere empor, herrlich schimmert sie im frischen Frühlingskleid, die Sonne gießt ihre segnenden Strahlen darüber aus.

Nun wird schon das erste Elbfeuerschiff sichtbar, der Lotse kommt an Bord, wir gleiten auf der breiten Elbe stromauf, auf beiden Seiten grüßen die lieblichen Ufer, die Häuser und Menschen, grüßt herrliches, üppiges Frühlingsgrün zu mir herüber. Bis sich aus dem Wasser eine mächtige schöne Stadt emportürmt, brandend und brausend uns ein Verkehr umgibt, da - „Fallen Anker“!

(Plüschow 1929, 223)

In diesem Modell der Expeditionsreisen fallen Fernweh und Heimweh durch das für die Expeditionsreisen typische kreisförmige Bewegungsmodell von Ausfahrt in die Fremde, Treffen auf Auslandsdeutsche und anschließende Rückkehr in die Heimat beinahe schon zusammen, womit Fremde ganz anders als im kolonialen Modell denkbar und artikulierbar wird.

Was sich historisch als Verheimatung der Fremde und als Expeditionsreise mit Rückkehr in die Heimat noch als ein Nacheinander darstellt, wird im Kino und Fernsehen der 1950er und frühen 1960er Jahre - mit Heimatfilmen wie Grün ist die Heide (1951) und Afrikafilmen wie Serengeti darf nicht sterben (1959) arbeitsteilig nebeneinandergestellt.

\section{Literatur}

Benninghoff-Lühl, Sibylle. Deutsche Kolonialromane 1884-1914 in ihrem Entstehungs- und Wirkungszusammenhang. Bremen: Übersee-Museum, 1983.

Bhabha, Homi K. Die Verortung der Kultur. Mit einem Vorwort von Elisabeth Bronfen. Übersetzt von Michael Schiffmann und Jürgen Freudl. Tübingen: Stauffenburg, 2000. 
Brehl, Medardus. „Vernichtung als Arbeit an der Kultur. Kolonialdiskurs, kulturelles Wissen und der Völkermord an den Herero“. Zeitschrift für Genozidforschung 2.2 (2000): 8-28.

Brehl, Medardus. „,Diese Schwarzen haben vor Gott und Menschen den Tod verdient‘. Der Völkermord an den Herero 1904 und seine zeitgenössische Legitimation“. Völkermord. Genozid und Kriegsverbrechen in der ersten Hälfte des 20. Jahrhunderts. Hg. Irmtrud Wojak und Susanne Meinl im Auftrag des Fritz Bauer Instituts. Frankfurt a. M.: Campus, 2004. 77-97.

Detering, Heinrich, und Kai Sina (Hg.). Kein Nobelpreis für Gustav Frenssen. Eine Fallstudie zu Moderne und Antimoderne. In Zusammenarbeit mit der Schleswig-Holsteinischen Landesbibliothek. Heide: Boysens, 2018.

Detering, Heinrich, und Kai Sina. „Poetik und Propaganda. Einführende Bemerkungen zu Gustav Frenssen“. Kein Nobelpreis für Gustav Frenssen. Eine Fallstudie zu Moderne und Antimoderne. In Zusammenarbeit mit der Schleswig-Holsteinischen Landesbibliothek. Hg. Heinrich Detering und Kai Sina. Heide: Boysens, 2018. 7-17.

Frenssen, Gustav. Jörn Uhl. Roman. Berlin: G. Grote’sche Verlagsbuchhandlung, 1901. Frenssen, Gustav. Hilligenlei. Roman. Berlin: G. Grote'sche Verlagsbuchhandlung, 1905.

Frenssen, Gustav. Peter Moors Fahrt nach Südwest. Ein Feldzugsbericht von Gustav Frenssen. Berlin: G. Grote'sche Verlagsbuchhandlung, 1906.

Frenssen, Gustav. „Wie ,Peter Moor“ entstanden ist“. G. Grotes Weihnachts-Almanach. Berlin: G. Grote'sche Verlagsbuchhandlung, 1909.1-5.

Freudenthal, René. „Die mangelhafte Frau. Stereotypen des Weiblichen, Hysterie und Wiedergängerschaft in Hans Grimms Novelle ,Als Grete aufhörte, ein Kind zu sein““. Acta Germanica. German Studies in Africa 38 (2010): 23-39.

Grimm, Hans. Südafrikanische Novellen. Lippoldsberg: Klosterhaus-Verlag, 1975.

Grün ist die Heide. Reg. Hans Deppe. Berolina, 1951.

Höpker, Lydia. Um Scholle und Leben. Schicksale einer deutschen Farmerin in Südwest-Afrika. Minden: Wilhelm Köhler, [1925?].

Horn, Peter. „Die Versuchung durch die barbarische Schönheit. Zu Hans Grimms ,farbigen“ Frauen“. Germanisch-Romanische Monatshefte 35 (1985): 317-341.

Jäger, Jens. „Colony as Heimat? The Formation of Colonial Identity in Germany around 1900“. German History 27.4 (2009): 467-489.

Jansen, Werner. „Gustav Frenssen siebenzig“. Gustav Frenssen-Almanach. Berlin: G. Grote'sche Verlagsbuchhandlung, 1933. 15-16 [zit. nach Joachim Warmbold. Deutsche Kolonialliteratur. Aspekte ihrer Geschichte, Eigenart und Wirkung, dargestellt am Beispiel Afrikas. Basel: Eigendruck, 1982. 97.].

Ketelsen, Uwe K. „Der Norden im Süden. Zu Friede H. Krazes ,Heim Neuland““. Tango del Norte. Festschrift für Walter Baumgartner. Hg. Cornelia Krüger und Frithjof Strauß (unter Mitwirkung von Dorothée Goetze). Greifswald: Ernst-Moritz-Arndt-Universität, 2006. 132-145.

Kiesel, Helmuth. „Moderne und Antimoderne. Gustav Frenssen im Kontext“. Kein Nobelpreis für Gustav Frenssen. Eine Fallstudie zu Moderne und Antimoderne. In Zusammenarbeit mit der Schleswig-Holsteinischen Landesbibliothek. Hg. Heinrich Detering und Kai Sina. Heide: Boysens, 2018. 21-42.

Kraze, Friede[rike] Henriette. Heim Neuland. Ein Roman von der Wasserkante und aus DeutschSüdwest. Stuttgart und Leipzig: Deutsche Verlagsanstalt, 1909.

Langbehn, Augst Julius. Rembrandt als Erzieher. Von einem Deutschen. 40. Aufl., Leipzig: Hirschfeld, 1892. 
Lienhard, Friedrich. Die Vorherrschaft Berlins. Literarische Anregungen. Berlin und Leipzig: Meyer, 1900.

Malherbe-Boucke, Julie. Südafrika von heute. Das Heimatland der Buren. Mit 16 Abbildungen und 1 Karte. Stuttgart: Strecker und Schröder, 1936.

Mehnert, Gottreich Hubertus. Mit Schwert \& Pflugschar in Sachsen und Südafrika. Anekdoten und Geschichten eines Südwester Pioniers. Überarbeitet von Bernd Kroemer. Windhoek: Glanz \& Gloria, 2007.

Meyn, Rolf. „Abstecher in die Kolonialliteratur. Gustav Frenssens ,Peter Moors Fahrt nach Südwest'“. Gustav Frenssen in seiner Zeit. Von der Massenliteratur im Kaiserreich zur Massenideologie im NS-Staat. Hg. Kai Dohnke und Dietrich Stein. Heide: Boyens u. Co., 1997. 316-346.

Parr, Rolf. „Nach Gustav Frenssens ,Peter Moor‘. Kolonialisten, Herero und deutsche Schutztruppe bei Hans Grimm und Uwe Timm“. Sprache im technischen Zeitalter 41.168 (2003): 395-410.

Parr, Rolf. Die Fremde als Heimat. Heimatkunst, Kolonialismus, Expeditionen. Konstanz: Konstanz University Press, 2014.

Plüschow, Gunter. Segelfahrt ins Wunderland. Im Reiche der Papageien und Guanakos. Berlin: Ullstein, 1926.

Plüschow, Gunter. Silberkondor über Feuerland. Mit Segelkutter und Flugzeug ins Reich meiner Träume. Berlin: Ullstein, 1929.

Serengeti darf nicht sterben. Reg. Bernhard Grzimek und Michael Grzimek. Okapia, 1959.

Warmbold, Joachim. Deutsche Kolonialliteratur. Aspekte ihrer Geschichte, Eigenart und Wirkung, dargestellt am Beispiel Afrikas. Basel: Eigendruck, 1982. 


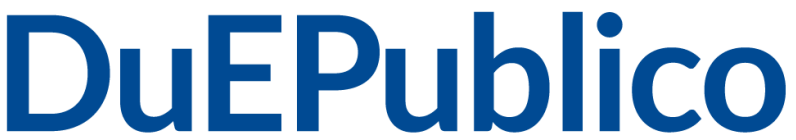

Duisburg-Essen Publications online offen im Denken

$\mathbf{U b} \mid \begin{gathered}\text { universitäts } \\ \text { bibliothek }\end{gathered}$

Dieser Text wird via DuEPublico, dem Dokumenten- und Publikationsserver der Universität Duisburg-Essen, zur Verfügung gestellt. Die hier veröffentlichte Version der E-Publikation kann von einer eventuell ebenfalls veröffentlichten Verlagsversion abweichen.

DOI: $\quad 10.1515 / 9783110650624-007$

URN: urn:nbn:de:hbz:464-20211011-095154-2

Parr, Rolf (2020) Koloniale Konstellationen von Heimat und Fremde. Wie Heimat und Fremde im Rückblick miteinander verschmelzen. In: Bönisch, Dana ; Runia, Jil ; Zehschnetzler, Hanna (Hrsg.). Heimat Revisited. (S. 127-144) Berlin ; Boston: De Gruyter. DOI: https://doi.org/10.1515/9783110650624-007

(C) 2020 Walter de Gruyter GmbH, Berlin/Boston Alle Rechte vorbehalten. 